\title{
Modelo para la evaluación del rol estratégico actual de una planta y despliegue de un plan para su mejora. Estudio empírico dentro de una red global de operaciones
}

\author{
Framework for assessing the current strategic factory role and deploying an \\ upgrading roadmap. An empirical study within a global operations network
}

\author{
Miguel Mediavilla', Ander Errasti² y Rosario Domingo' \\ 'Dpto. de Ingeniería de Construcción y Fabricación. UNED. Madrid. \\ 2Dpto.de Organización Industrial.TECNUN. Universidad de Navarra. San Sebastián \\ miguel.mediavilla@gmail.com aerrasti@ceit.es rdomingo@ind.uned.es
}

Fecha de recepción: 12-8-2010

Fecha de aceptación: 20-10-2010

\begin{abstract}
Resumen: Una economía globalizada necesita respuestas eficientes para servir a mercados globales. Empresas de todos los tamaños tienen dificultades para gestionar sus complejas redes globales de operaciones; sin embargo, la bibliografía relacionada proporciona pocosmodelos para diseñar/reestructurar estas redes. Tomando como base el modelo de Ferdows para el análisis del rol de planta dentro de redes globales de operaciones, el objetivo de este artículo es doble: I) proporcionar un estudio empírico de la aplicación de dicho modelo, y 2) proponer un modelo sistemático y priorizado que eleve el rol estratégico de una planta dentro de su red de operaciones.
\end{abstract}

Palabras clave: Red global de operaciones, rol estratégico de planta, estudio de caso.

Abstract: A globalised economy needs an efficient respond to global markets by globally sourcing, manufacturing and supplying. All size companies face difficulties when managing complex global operations networks, but meanwhile the related literature contains few models to design/restructure those. Based on the model of Ferdows for factory role analysis within global operations network, the aim of this paper is twofold: I) to provide empirical testing the mentioned model and 2) to present a framework for a focused and systematic upgrade of a given factory role within its operations network.

Keywords: global operations network, strategic factory role, case study.

\section{Introducción}

En los recientes años de expansión económica, caracterizados por grandes cambios macroeconómicos y un entorno competitivo cada más globalizado, la internacionalización de las operaciones se ha convertido en una tendencia común entre las empresas -principalmente multinacionales pero también en pequeñas y medianas empresas (Corti et al., 2009). Con la aparición defuentes de suministro y fabricaciónglobales, y mercados globalizados, el diseño de redes de operaciones tiene que abarcar múltiples regiones que cada vez son más extensas, lo cual obliga a afrontar redes con mayor complejidad. Aun así, la literatura existente sobre cómo diseñar o reestructurar una red global de operaciones es escasa y los aspectos de estudio están muy dispersos (Corti et al., 2009).
Ferdows (1989, 1997) propone un interesante modelo para clasificar, dentro de una red global de operaciones, cualquier planta en una serie de roles predefinidos -basándose en el grado de competencias y en el factor estratégico de su localización (Figura I).

Los roles fábrica dentro de una red productiva quedan así definidos, pero el despliegue operativo de sus características, sin embargo, no ha sido explícitamente desarrollado (Verecke y Van Dierdonck, 2002). Por ende, cualquier intento para elevar el rol de fábrica resulta difícil de formular, desplegar y priorizar.

Este artículo se basará en la aplicación del modelo de Ferdows para el análisis de una red de operaciones ya existente, mediante la evaluación de 34 plantas productivas de una compañía con ámbito de operación global, y lo extenderá al desarrollar un modelo 
Figura I

Matriz de posicionamiento de los roles de planta según nivel de competencias y factor estratégico para la localización de planta (Ferdows, 1997)

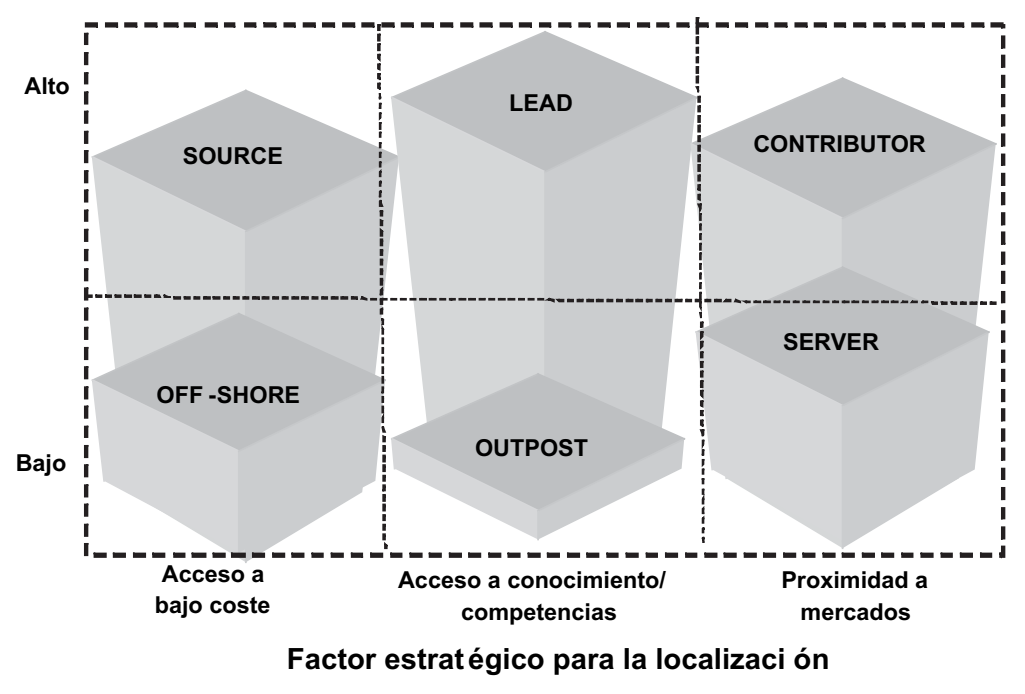

Tabla I

Características de los roles de planta propuestos por Ferdows $(1989,1997)$

\begin{tabular}{|c|c|}
\hline $\begin{array}{l}\text { ROL DE PLANTA } \\
\text { PRODUCTIVA }\end{array}$ & CARACTERÍSTICAS \\
\hline OFF-SHORE & $\begin{array}{l}\text { Una fábrica «offshore» se establece para fabricar productos específicos a bajo coste, que suelen posterior- } \\
\text { mente exportarse para completarlos o venderlos. Las inversiones en instalaciones y recursos para su ges- } \\
\text { tión se suelen mantener al mínimo necesario. No disponen de grandes capacidades de desarrollo de pro- } \\
\text { ducto o ingeniería. Rara vez disponen de capacidad de decisión sobre proveedores o en la negociación de } \\
\text { precios de compra. El área económica básicamente reporta datos a la gerencia en la central. La logística de } \\
\text { distribución es simple y limitada, y fuera del control de la dirección de fábrica. }\end{array}$ \\
\hline SOURCE & $\begin{array}{l}\text { El objetivo principal de establecer una fábrica «source» es la búsqueda del bajo coste, pero su rol estraté- } \\
\text { gico es más amplio que una fábrica «offshore». Su equipo de gestión tiene una mayor responsabilidad so- } \\
\text { bre el aprovisionamiento (incluyendo la selección de proveedores), planificación de producción, logística de } \\
\text { distribución, mejoras en proceso, así como la personalización/rediseño de productos. Una fábrica «source» } \\
\text { tiene la misma capacidad para producir un producto o componente como la mejor fábrica de la red. Las fá- } \\
\text { bricas «source» tienden a localizarse en países de bajo coste laboral, infraestructuras desarrolladas y don- } \\
\text { de se encuentren trabajadores formados. }\end{array}$ \\
\hline OUTPOST & $\begin{array}{l}\text { La tarea principal de una fábrica «outpost» es recopilar información, dado que suelen situarse en áreas don- } \\
\text { de existen proveedores avanzados, competidores, centros de investigación o clientes. Dado que, obviamente, } \\
\text { toda fábrica debe producir productos y servir a mercados, las fábricas «outpost» tienen un rol estratégico } \\
\text { secundario como fábrica «server» o «offshore» — por ejemplo. }\end{array}$ \\
\hline LEAD & $\begin{array}{l}\text { Una fábrica «lead» crea nuevos procesos, productos y tecnologías para toda la compañía. Este tipo de plan- } \\
\text { ta industrial potencia las competencias locales y los recursos tecnológicos no sólo para adquirir informa- } \\
\text { ción para la compañía, sino para transformar la información que recoge en utilizaciones reales en produc- } \\
\text { tos o procesos. Su equipo de gestión tiene una participación decisiva en la elección de proveedores } \\
\text { estratégicos y participan frecuentemente en desarrollos conjuntos con estos. Muchos de los empleados de } \\
\text { las fábricas «lead» están en contacto directo con clientes finales, proveedores de instalaciones y centros de } \\
\text { investigación. En estas plantas se suelen iniciar habitualmente innovaciones. }\end{array}$ \\
\hline
\end{tabular}


Tabla 1

Características de los roles de planta propuestos por Ferdows (1989, 1997) (continuación)

\begin{tabular}{|c|l|}
\hline $\begin{array}{c}\text { ROL DE PLANTA } \\
\text { PRODUCTIVA }\end{array}$ & \multicolumn{1}{c|}{ CARACTERísTICAS } \\
\hline SERVER & $\begin{array}{l}\text { Una fábrica «server» abastece mercados nacionales o regionales específicos. Es habitual que estas plantas } \\
\text { proporcionen ventajas para evitar barreras arancelarias o que reduzcan impuestos, costes logísticos o la de- } \\
\text { pendencia a fluctuaciones de algún tipo de cambio extranjero. A pesar de tener algo más de autonomía que } \\
\text { las plantas «offshore» para realizar cambios menores en productos y métodos de fabricación -para adap- } \\
\text { tar el producto al mercado asignado-, su autoridad y competencias están muy limitadas. }\end{array}$ \\
\hline \multirow{6}{*}{ CONTRIBUTOR } & $\begin{array}{l}\text { Una fábrica «contributor» también abastece a un mercado nacional o regional específico, pero sus respon- } \\
\text { sabilidades abarcan también la ingeniería de producto y producto, así como a la elección y desarrollo de } \\
\text { proveedores. Una fábrica «contributor» compite internamente con las plantas situadas en el país de origen } \\
\text { de la compañía para servir de área piloto para nuevas tecnologías de proceso, sistemas informáticos y pro- } \\
\text { ductos. Tienen su propio área de desarrollo de producto, ingeniería de procesos y producción. Una fábrica } \\
\text { «contributor» suele también tomar parte en las decisiones referidas a aprovisionamientos y participa en la } \\
\text { elección de proveedores clave para la compañía. }\end{array}$ \\
\hline
\end{tabular}

que permita, de una manera sistemática y priorizada, desplegar una plan de mejora que facilite la elevación gradual del rol de una planta dentro de su red de operaciones.

\section{Revisión bibliográfica}

La estrategia de operaciones se define como el conjunto global de decisiones que moldean las capacidades a largo plazo de cualquier tipo de operación y su contribución a la estrategia general de negocio mediante la reconciliación de las demandas del mercado con los recursos de operaciones (Slack y Lewis, 2002).

Esta estrategia se debe reflejar en el diseño de las redes de operaciones, que básicamente trata de dónde localizar las fuentes de aprovisionamiento, fabricación y distribución, así como el despliegue de dichas operaciones (es decir, quién debería proveer a quién y la planificación de instalaciones). En este proceso, el impacto cuantitativo de factores como los costes fijos y variables de producción o las instalaciones de distribución, inventario, transporte y otros costes logísticos deben ser considerados (Lee y Silvermann, 2008). El mismo procedimiento debe seguirse para la racionalización o reestructuración de una red de operaciones ya existente (Vereecke y Van Dierdonk, 2002).

Un enfoque común para la internacionalización que persigue reducir costes y aumentar la competitividad a corto plazo, es la creación y gestión de plantas productivas en países foráneos para únicamente beneficiarse de ventajas arancelarias, costes laborales menores, subvenciones, y menores costes logísticos.
Como resultado (Ferdows, 1997), estas plantas poseen un número limitado de responsabilidades, capacidad de decisión, participación en la red de operaciones, y recursos asignados.

Otras compañías tienen mayores expectativas en sus plantas foráneas $y$, por lo tanto, tratan de conseguir mucho más de ellas. Este acercamiento proporciona no sólo acceso a las ya mencionadas ventajas dereducción de costes, sino también una red de producción y distribución globalcon mayor proximidad a potenciales mercados,con acceso cercano a clientes, proveedores, o a trabajadores con competencias específicas, motivados o con ciertas habilidades. Estas plantas suelen tener una mayor capacidad de gestión y autonomía en la toma de decisiones que la simple administración operativa de la producción, como por ejemplo: desarrollo de producto y proceso, capacidad de decisión en aprovisionamientos y compras, servicio de asistencia técnica, etc. (Ferdows, 1997).

Sin embargo conviene no limitarse a considerar cada planta de manera aislada dentro de la red productiva, ya que la coordinación de la red de plantas puede ser fuente de ventaja competitiva (Shi y Gregory, 2005). Por tanto el evolucionar de una planta con poca autonomía y con muy baja interacción dentro de la red global, para introducirla e integrarla dentro de una red global de operaciones, permite beneficiarse de sinergias entre plantas, reducir los costes, aumentar la fiabilidad de las entregas, y acortar la curva de aprendizaje de otros miembros de la red de operaciones (Flaherty, 1986).

Si los directivos de las empresas no consideran la fabricación como una fuente de ventaja competitiva, 
probablemente establezcan fábricas foráneas con un bajo perfil estratégico. Por el contrario, si se considera la fabricación como una fuente principal de ventaja competitiva, entonces las plantas foráneas proporcionarán a su empresa una mayor cuota de mercado y mayores rentabilidades (Ferdows, 1997). Además, debido a la competencia cada vez más globalizada, la distancia entre las compañías que consideran sus plantas extranjeras como fuente de ventaja competitiva y aquellas que no lo hacenaumenta constantemente (Ferdows, 1997).

La literatura sobre la gestión de operaciones recoge diversos modelos que tratan sobre el análisis y rendimiento de la cadena de suministro (ver lista de modelos en, por ejemplo, Netland et al. (2007) o Corti et al. (2009)) pero únicamente unos pocos cambian su enfoque desde la cadena de suministro y lo amplían a un acercamiento desde una perspectiva de la cadena de valor - como es definida por Porter (1985).

Ferdows ( 1989 y 1997) abarca de forma implícita la cadena de valor y la idea de una red internacional de producción, especialmente con la introducción del concepto de fábrica «lead»: una planta que contribuye a la estrategia de la compañía, por ejemplo, desarrollando nuevos procesos, productos o tecnologías,contacto con clientes, proveedores, etc. Y estas capacidades podrían ser compartidas con otras plantas de la red. Otro trabajo que merece una mención es el concepto de «Manufacturing Value Chain» de Singh Srai y Shi (2008), que proporciona una perspectiva de la cadena de valor que podría relacionarse con el concepto de rol de fábrica de Ferdows extendido no sólo a la planta como sujeto de análisis, sino a la propia red de plantas. Sin embargo, a pesar que Ferdows es un modelo de referencia que está logrando reconocimiento académico en el campo de la internacionalización de operaciones - (como por ejemplo Vereecke y Van Dierdonck, 2002; Fusco y Spring, 2003; Meijboom y Voordijk, 2003; Maritan et al., 2004; Vereecke et al., 2006; Corti et al., 2009; Feldmann y Olhager, 2009) — y este modelo es fácilmente reconocible en la práctica por directivos de empresas, todavía existen pocas evidencias empíricas de la aplicación del modelo de Ferdows.

\section{I. Entorno y dinámica del rol estratégico de planta}

La ampliación de la Unión Europea (UE) ha creado un espacio económico más extenso y unificado que proporciona un mayor acceso a países de bajo cos- te laboral y nuevos mercados a las compañías multinacionales (Agrawal et al., 2003). Igualmente ocurre con otros países con bajos costes laborales (Turquía, Rusia u otros países del Este de Europa) con una proximidad geográfica a la Unión Europea. En consecuencia muchas empresas han expandido sus operaciones y redefinido los roles de sus unidades organizativas situadas en los diferentes países (Radovilsky, 1994; Schmenner, 1997; Corti et al., 2006). Algunas compañías han empezado a reaccionar con nuevas estrategias europeas, como es el movimiento de factorías a países de bajo coste, consolidando y localizando sus centros logísticos en el este de Europa y consolidando sus organizaciones regionales para convertirlas en unidades operacionales para toda Europa (Wong y Gubi, 2007).

Todos estos cambios requieren de nuevo conocimiento en la gestión de operaciones en una Europa cada vez más expandida (Prasad et al., 200 I), situando a las compañías de Europa Occidental en una situación paradójica en lo que concierne a sus instalaciones productivas en países con altos costes laborales. Muchas de las nuevas estrategias en compañías europeas se orientan a una redefinición de los roles de sus fábricas y centros de ventas en el extranjero, así como de sus oficinas centrales. Se observa un comportamiento similar en multinacionales estadounidenses y de Europa Occidental con subsidiarias en otrospaíses de bajo coste fuera de la UE (Ghemawat, 2010).

Sin embargo, el inherente desarrollo social y económico de los países en los que se localizan fabricas de bajo coste («offshore») hace que actualmente sea cada vez más difícil mantener una clasificación de países de alto coste (High-cost countries o HCC) y de bajo coste (Low- cost countries o LCC). Cualquier cambio en el entorno, como por ejemplo, factores macroeconómicos (inflación, etc.), socioeconómicos (leyes laborales, costes laborales, etc.), o la inestabilidad política, podrían provocar una rápida degradación del rol hasta entonces alcanzado por una planta foránea y por ende, dañar sus competitividad. Estas condiciones dinámicas provocan la necesidad de desarrollar diferentes capacidades o competencias operacionales, haciendo del dinamismo una constante en el papel estratégico de cada rol dentro de su red global de operaciones.

El diseño de redes internacionales de fabricación, por tanto, deben integrar la evaluación de las capacidades de adoptar ese dinamismo (Sweeney et al., 2007), como ocurre en cualquier racionalización o reestructuración de redes de operaciones. Estas capaci- 
dades dinámicas son definidas porTeece et al. (1997) como «la capacidad de lograr nuevas formas de ventaja competitiva para enfatizar dos aspectos clave que no fueron foco de atención en perspectivas estratégicas anteriores». El término «dinámico» se refiere a la capacidad de renovar las competencias para ir siendo coherente con los cambios en el entorno del negocio. El término «capacidades» enfatiza el rol clave de una gestión estratégica en adaptar, integrar y reconfigurar adecuadamente las habilidades internas y externas, recursos y competencias funcionales para adaptarse a los requerimientos de un entorno cambiante.

\section{Preguntas de investigación y objetivos}

La revisión bibliográfica confirma que, aún cuando algunos estudios han prestado atención a los roles de la fábrica dentro de una red de fabricación (Ferdows, 1989 y 1997; Vokurka y Davis, 2004), el modelo desarrollado por Ferdows ( 1989 y 1997)dispone de pocas evidencias empíricas más allá del estudio de caso (Vereecke y Van Dierdonck, 2002) y por lo tanto, se podría enriquecer con aportaciones que desde lainvestigación empírica se puedan realizar (Chakravarty et al., 1997; Vereecke y Van Dierdonck, 2002; Corti et al, 2009).

Asimismo, parece clara la necesidad de extender la investigación para poder entender cómo coordinar las operaciones de unidades individuales de producción pertenecientes a una red de fabricación (Dubois et al., 1993; Shi y Gregory, 1998 y 2005). La manera de cómo lograr que el rendimiento de una red global sea mayor que la suma de cada una de sus partes no es un aspecto bien entendido (Rudberg, 2004) en un contexto económico altamente dinámico y un entorno competitivo globalizado (Corti et al., 2006). Concretamente se necesitan modelos y técnicas que ayuden en la práctica a formular y desarrollar estrategias de operaciones (Slack y Lewis, 2002) cuando se afronten reestructuraciones de redes ya existentes (Vereecke y Van Dierdonck, 2002) con objeto de renovar las capacidades y competencias de las fábricas (Teece et al., 1997; Sweeney et al., 2007).

Este artículo explora la aplicación empírica del modelo de Ferdows y extiende su aplicación al proponer un modelo que despliegue el cómo lograr una mejora del rol estratégico de una planta dentro de una red global de operaciones - basado en la identificación de las debilidades: es decir, se abarca mucho más que una mera identificación empírica del análisis del rol actual de una planta.

\section{Modelo propuesto para evaluar/mejorar el rol de planta}

Si las competencias de una planta no son reforzadas para conseguir una mejora del rol estratégico, normalmente las plantas menos exitosas podrían desaparecer de la red debido a la presión competitiva para reducir costes y concentrar el volumen de producción en un número más reducido de plantas (De Meyer y Vereecke, 1996). Por lo tanto, las compañías deben rediseñar y reconfigurar sus cadenas de suministro multilocalizadas. Cualquier iniciativa de rediseño citada puede efectuarse de manera global (red completa) o parcial (unidades de producción), lo cual implicará un enfoque diferente en su planteamiento:

- Acercamiento Top-down: las compañías se ven obligadas a racionalizar y reestructurar su red de operaciones para adaptarse a condiciones cambiantes y desarrollar diversas capacidades operacionales: reforzando su ventaja competitiva para responder a las necesidades de sus clientes. El afrontar estos retos implicará decisiones que determinarán para la red qué competencias/capacidades se deben conservar, qué nuevas competencias/capacidades se quieren desarrollar y qué actividades es aconsejable externalizar.

- Acercamiento Bottom-up: las fábricas son forzadas a desarrollar sus competencias para así elevar su rol estratégico y, por ende, no verse forzadas a desaparecer de la red global de operaciones de la compañía.

Se considera que el rediseño de las operaciones se debe realizar alineándolo en tres niveles, bajo una perspectiva de contingencia estratégica (Defee y Stank, 2005): I) Entorno de la cadena de suministro, 2) Estrategia de operacionesy 3) Eficiencia. La competencia de la cadena de suministro de una nación es entendida y definida como la capacidad que posee un país para mantener prácticas excelentes en la gestión de la cadena de suministro, instituciones e infraestructuras, desde una perspectiva de cadena de suministro y logística (Kinra et al., 2007). Gobbo (20007) realiza una revisión bibliográfica sobre la priorización competitiva en función de la estrategia de operaciones y el rendimiento al llevar a cabo un rediseño de una red de operaciones. Esta se resumeen los siguientes indicadores: Coste (C), Calidad (Q), Velocidad (S), Flexibilidad (F), Innovación (I) y Fiabilidad (R) - que podrían ser utilizados a posteriori como base de medida para ciclos de mejora. Los autores de este trabajo de investigación han centrado su modelo para cubrir estos indicadores (com- 
Figura 2

Esquema del modelo: pilares, métodos y herramientas

\begin{tabular}{|l|}
\hline \multicolumn{1}{|c|}{$\begin{array}{c}\text { Producción orientada } \\
\text { a la demanda }\end{array}$} \\
\hline \multicolumn{1}{|c|}{ Principio -Pull } \\
\hline - Análisis -ABC/XYZ \\
- IIT/IIS \\
- Kanban/Supermarket \\
- Milk run \\
- Vendor Managed inventory \\
(VMI) \\
\hline \multicolumn{1}{c|}{ Takt -time } \\
\hline - Producción nivelada y suavi- \\
zada \\
- Cambio rápido (SMED) \\
- Diseño del flujo de valor \\
(Value stream mapping) \\
Gestión de suministros \\
\hline - Make or Buy \\
- Selección y desarrollo de pro- \\
veedores \\
- Integración de proveedores \\
- Gestión de proveedores \\
- Tool management \\
- Logística de aprovisionamien- \\
to \\
\hline
\end{tabular}

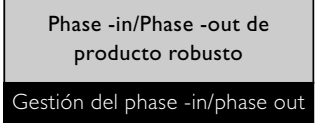

- Lanzamiento de variantes$$
\text { (1) }
$$

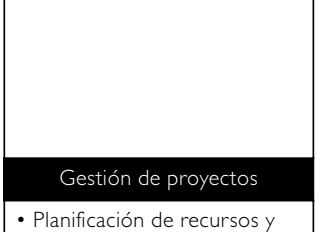

- Planificación de recursos y

tiempos

- Gestión del riesgo y escalado

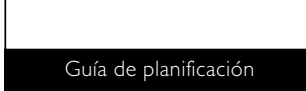

- Planificación de procesos

- Planificación de fábricas

- Planificación de localizaciones

- Ergonomía y seguridad

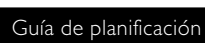

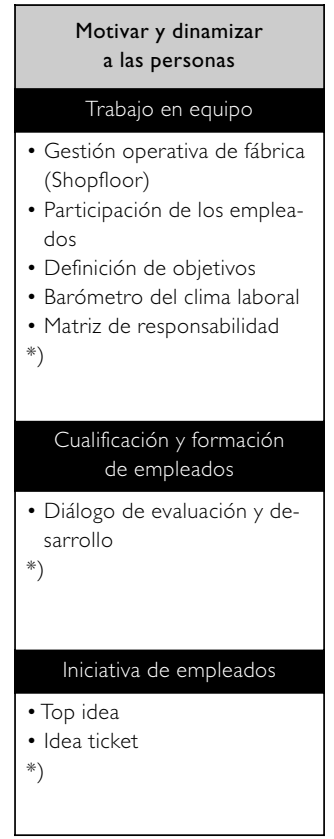

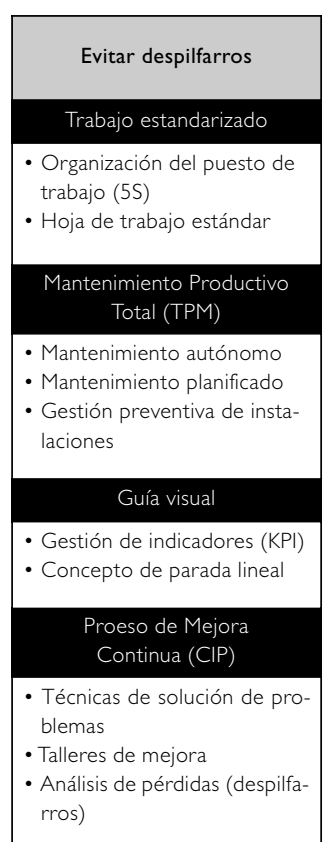

rros)

Estandarización y Sostenibilidad

plementándolos con, por ejemplo, motivación (M)) y poder medir el rendimiento de una planta productiva, uniéndolo a la identificación del rol estratégico de planta actual: este modelo se compone de cinco pilares, los cuales están desplegados en métodos y herramientas (Figura 2). Los cinco principios del pensamiento Lean (producción ajustada) y su enfoque del flujo de valor (Value Stream) (Womack et al., 1990; Womack y Jones, 1996) han sido una base importante en la definición del modelo.

El modelo se divide en 5 pilares que cubren transversalmente —y en mayor o menor grado- los indicadores citados (Tabla 2) y que se basan en los tres procesos principales de una empresa manufacturera propuestos por Womack y Jones (1996):

- Proceso «Pedido —Entrega»: cubierto por el l er pilar

- Proceso «Diseño de producto -lanzamiento en serie»: cubierto por el $2^{\circ}$ pilar y $5^{\circ}$ pilar.

- Proceso «físico de transformación»: cubierto por el $4^{\circ}$ y $5^{\circ}$ pilar.

*el tercer pilar soporta los tres procesos principales y los demás procesos secundarios.
Los métodos y herramientas propuestas son el despliegue de cada pilar y persiguen apoyar el logro de la visión definida en cada pilar (Tabla 2); esta visión es semejante a la definición de «proceso perfecto» definido por los autores dentro del pensamiento Lean (Ohno, 1988; Womack y Jones, 1996).

Los indicadores citados están específicamente definidos en el cuestionario de evaluación, en las preguntas asignadas a pilares, herramientas y/o métodos (véase Tabla 2).

El esquema ha sido creado considerando la capacidad de transferir cualquier sistema de gestión a otros países -a pesar de las diferencias culturales-, dado que el desarrollo de la teoría de gestión se basa en lógica general (Harbison y Myers, 1989). Los sistemas de gestión específicos se relacionan a menudo con las prácticas excelentes y son aplicables en cualquier nación (Koontz 1969; Kono, 1992). Sin embargo, se necesita un acercamiento de contingencia (Beechler y Yang, 1994), donde una buena adecuación (estrategia, política y prácticas en el contexto) posibilitarán el alcanzar un buen rendimiento. Algunos autores afirman que los sistemas de gestión no son ni rechazados ni aceptados, sino que se crean hibridaciones junto a los sistemas de gestión locales (Koontz, 1969; 
Tabla 2

Indicadores incorporados en el cuestionario de evaluación

\begin{tabular}{|c|c|c|c|c|c|}
\hline & $\begin{array}{l}\text { Producción orientada } \\
\text { a la demanda }\end{array}$ & $\begin{array}{l}\text { Phase-in/phase-out } \\
\text { de producto robusto }\end{array}$ & $\begin{array}{l}\text { Motivar y dinamizar } \\
\text { a las personas }\end{array}$ & $\begin{array}{l}\text { Evitar defectos } \\
\text { (cero defectos) }\end{array}$ & Evitar despilfarros \\
\hline Visión & Flujo continuo & $\begin{array}{l}\text { Cero desvíos frente } \\
\text { al plan en nuevas } \\
\text { introducciones }\end{array}$ & $\begin{array}{c}100 \% \\
\text { de los empleados } \\
\text { motivados }\end{array}$ & Cero defectos & $\begin{array}{c}100 \% \\
\text { de valor añadido } \\
\text { en los procesos }\end{array}$ \\
\hline $\operatorname{COSTE}(\mathrm{C})$ & $\mathbf{x}$ & $\mathbf{x}$ & & $\mathbf{x}$ & $\mathbf{x}$ \\
\hline CALIDAD (Q) & & & & $\mathbf{x}$ & \\
\hline VELOCIDAD (S) & $\mathbf{x}$ & $\mathbf{x}$ & & & \\
\hline FLEXIBILIDAD (F) & $\mathbf{x}$ & & $\mathbf{x}$ & & \\
\hline INNOVACIÓN (I) & & $\mathbf{x}$ & $\mathbf{x}$ & & $\mathbf{x}$ \\
\hline FIABILIDAD (F) & $\mathbf{x}$ & $\mathbf{x}$ & & $\mathbf{x}$ & $\mathbf{x}$ \\
\hline MOTIVACIÓN (M) & & & $\mathbf{x}$ & & $\mathbf{x}$ \\
\hline
\end{tabular}

Abo, 1994; Kumon y Abo, 2004) y que este grado de hibridación viene determinado por circunstancias locales tales como, por ejemplo, cultura, actitud y ética de los trabajadores, educación y formación, sindicatos, practicas de gestión locales, dificultades de comunicación, consideraciones económicas, industria y sector (Yokozawa et al., 2007). Si las circunstancias locales (Yokozawa et al., 2007) y el sistema de gestión son tomadas en cuenta, la evaluación del rol estratégico de planta y su formulación podrían ayudar fusionando el entorno, la estrategia de operaciones, las prácticas y el rendimiento a lograr, así como proporcionando un plan de mejora para ese propósito.

\section{Estudio de caso: Recopilación de datos y chequeo del modelo}

El estudio de caso se desarrolla en una compañía multinacional de electrodomésticos dedicada al diseño, fabricación y distribución, que facturó más de 8.000 millones de Euros en 2009 y es una de las compañías líder del sector. La empresa posee más de 40 fábricas en Europa, EEUU, Latinoamérica y Asia, con un número de trabajadores aproximado de 40.000. El modelo desarrollado ha sido implementado en la mayor parte de 34 plantas (7 países) desde comienzos de 2007, para lo cual se han creado departamentos centrales y en las divisiones de producto, y se ha nombrado a un coordinador en cada planta. Los departamentos centrales y de las divisiones de producto se han responsabilizado de comunicar y transferir las buenas prácticas existentes, así como el ofrecer formación, asesoría y consultoría práctica en las plantas. Los coordinadores en planta se han responsabilizado de dinamizar y empujar la implementación del modelo.

Las plantas han sido evaluado en 2008 y 2009 mediante auditorias in situ (realizadas por al menos 2 personas) basándose en un cuestionario de revisión (las fábricas que han comenzado la implementación del modelo en 2008 han sido primeramente evaluadas en el 2009). El resultado final y la puntuación obtenida ha sido extensamente chequeado mediante un panel Delphi (Linstone y Turoff, I 975; Turoff y Hitz, 1996) compuesto por equipo directivo de la planta así como las sedes centrales para reforzar la validez de los resultados.

El cuestionario tiene 400 preguntas (máximo 1000 puntos en total y 200 puntos por pilar) referidas a cuántos y en qué grado de extensión han sido implementados los métodos y herramientas previamente mencionados. Las preguntas se puntúan con el siguiente criterio (en función del alcance definido): 0 (no hay nada planificado ni implementado), I (planificado), 2 (área piloto), 3 (>50\% del área de aplicación), 4 (totalmente implementado en todo el área de aplicación).

El cuestionario inicial -basado únicamente en preguntas con respuesta cuantitativa- fue extendido, 
Figura 3

Evaluación de las fábricas con el cuestionario y clasificación

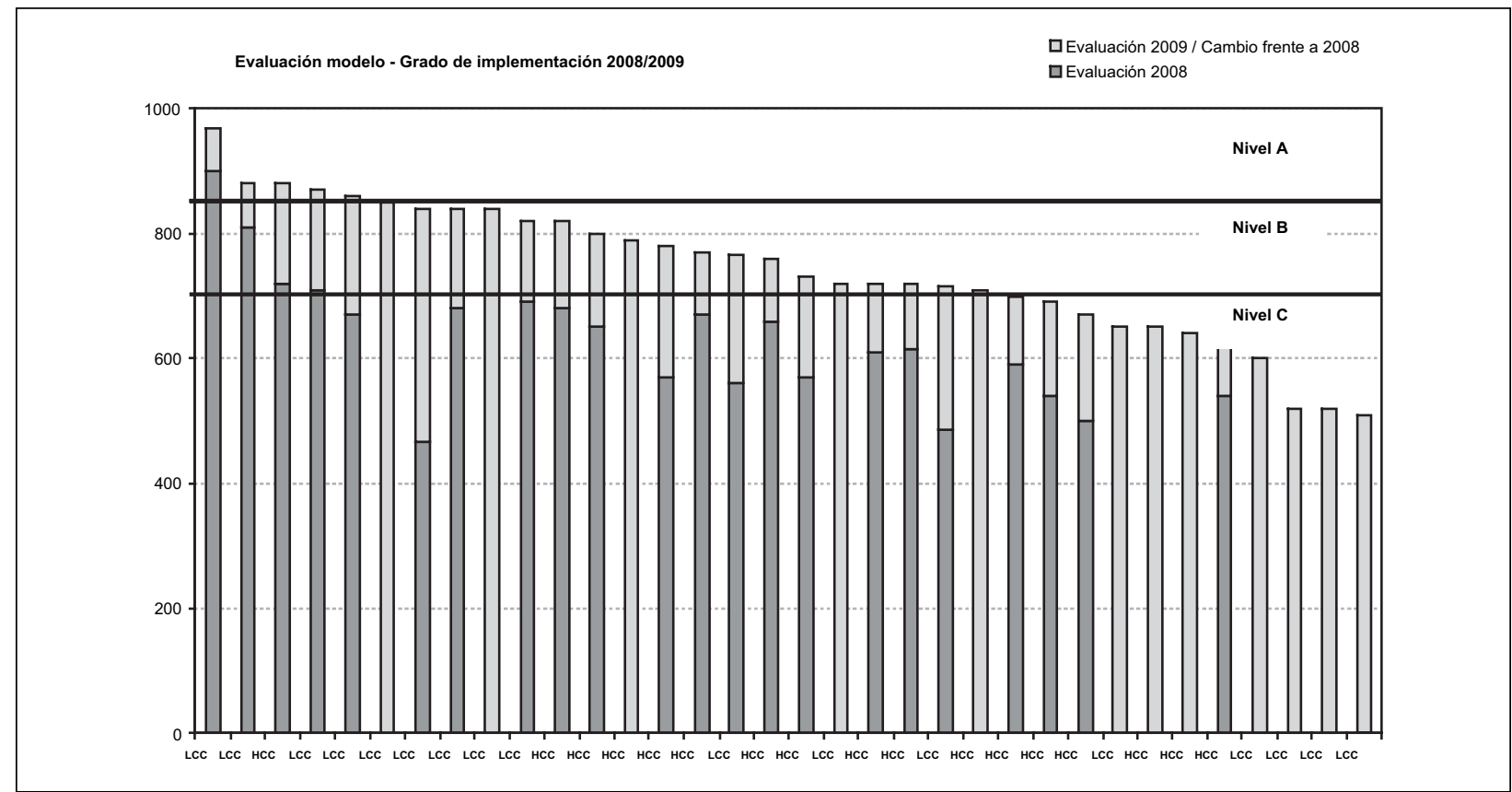

haciendo que cada método y herramienta fuesen complementados con varias cuestiones cualitativas que se respondan con «no», «parcialmente» o «sí»»; estas preguntas han proporcionado un mejor criterio al evaluar las cuestiones cuantitativas inicialmente formuladas.

Basándose en el resultado final del cuestionario se establece la siguiente clasificación de plantas (resultados 2009): A (>850 puntos): 6 fábricas; B (700-850 puntos): 18 fábricas; $C$ (menos de 700 puntos): 10 fábricas.

Resultado de la clasificación según el modelo desarrollado, así como bajo el modelo de Ferdows:

- El rol de fábrica ha sido determinado en los paneles Delphi mediante entrevistas personales individuales y utilizando un cuestionario estructurado. Las conclusiones y aportaciones fueron trasladadas a los entrevistados para su revisión y aceptación.

- Excepto una planta «lead» clasificada como A, el resto de plantas $A$ fueron inicialmente establecidas en países de bajo coste (LCC) como fábricas «offshore»y posteriormente «source». Se observa que alguna de las fábricas de mayor puntación está empezando a mejorar su rol para convertirse en fábricas 《contributor» - habiendo mejorado sus resultados en ese tiempo. Dos de estas fábricas pueden ser valoradas como plantas «lead» para ciertas competencias específicas (los métodos con mayor valoración).

- La mayoría de plantas catalogadas como B se encuentran localizadas en países de alto coste (mayoritariamente Europa occidental) e incluyen a todas las fábricas «lead» excepto una la planta ya mencionada. La evaluación de las fábricas «lead» ha mejorado considerablemente de 2008 a 2009 pero todavía no ha llegado a ser excelente.

- Las fábricas C son mayormente fábricas «off-shore» O «source» recientemente establecidas. Algunasfábricas $C$ localizadas en países de alto coste son consideradas «contributor», a pesar de lo cual se encuentran actualmente bajo severos programas de mejora de productividad y competitividad (áreas de trabajo basadas en los pilares del modelo con menor puntuación).

Valoraciones externas del modelo:

- La fábrica con mayor puntuación en 2009 fue también galardonada con el premio japonés (JIPM) de excelencia en TPM (categoría A).

- Las mejoras cuantitativas en materiales, costes laborares y gastos generales directamente vinculados con la implementación del esquema se cuan- 
tificaron alrededor de 3 puntos porcentuales del EBIT anual (Beneficios antes de intereses e impuestos: indicador que representa el margen o resultado operativo de explotación de la empresa antes de intereses y impuesto de sociedades. Es una medida de la rentablidad de la empresa y sirve para la valoración de la misma) en el periodo 2007-2009.

- Otros beneficios indirectos (flexibilidad, motivación, estandarización) han sido explícitamente reconocidos por panel Delphi de expertos.

\section{Conclusiones y Limitaciones}

Las principales conclusiones relacionadas el propósito de este trabajo son:

- El modelo propuesto sirve como primera herramienta de análisis para evaluar el rol estratégico de una planta.

- La evaluación del esquema prioriza aspectos de mejora, los cuales posteriormente podrían impactar en la mejora del rol de fábrica.

- Se ha probado que la implantación del esquema ayuda a mejorar el rendimiento económico de cada planta mediante, principalmente, la mejora de la eficiencia. La mejora del rendimiento económico se ha identificado en cada planta —más allá del país de aplicación.

- El cuestionario de evaluación basado en el grado de implementación (cuantos métodos/herramientas y en qué extensión) es altamente eficiente en los 304 años de aplicación del modelo.

Las limitaciones y líneas futuras de investigación identificadas para el modelo son:

- Toda vez que las plantas hayan alcanzado un alto de implementación de los métodos y herramientas propuestas por el modelo, sería necesario desarrollar un cuestionario de evaluación que midiera el grado de madurez (cuán bien y con qué rendimiento) para tener una valoración directa del rendimiento operativo. Se han realizado tres experiencias de evaluación de plantas clasificadas como «B» con una primera versión de un cuestionario de madurez -adicional al cuestionario de implementación ya explicado-y que confirma la necesidad de profundizar en cues- tionarios que evalúen la calidad de la implementación tras la fase inicial de implementación.

- El esquema no considera explícitamente la contribución original de las fábricas «lead», las cuales obtienen valoraciones escasas en ciertas cuestiones y que pueden deberse a la falta de experiencia en ciertos aspectos (principalmente en áreas que van más allá de la cadena de suministro y que forman parte de la cadena de valor), dado el carácter pionera que deben adoptar estas plantas «lead». El resto de plantas se benefician, por tanto,de las experiencias ya vividas en las plantas «lead».

- El modelo podría extenderse o ser más específico con algunos aspectos de la cadena de valor, los cuales también influyen en el rol estratégico de fábrica - y que principalmente suelen asumir inicialmente las plantas «lead»: por ejemplo, innovación en producto/proceso, gestión de la cartera de producto, gestión estratégica de proveedores, flexibilidad hacía diferentes regiones.

\section{Bibliografía}

ABO, T.(1994), Hybrid factory: The Japanese production system in the United States, Oxford University Press, New York.

AGRAWAL,V., FARRELL, D. y REMES, J.(2003), Offshoring and beyond, McKinsey Quarterly, 3, 24-35.

BEECHLER, S. y YANG, J. Z. (1994), The transfer of Japanese-style management to American subsidiaries: Contingencies, constraints, and competencies, Journal of International Business Studies, Vol.25, No.3, pp. 467-49I.

CORTI, D., EGAÑA, M.M. y ERRASTI, A. (2009), Challenges for off-shored operations: findings from a comparative multi-case study analysis of Italian and Spanish companies,. Proceedings I6th annual EurOMA Conference, Gothenburg.

CORTI, D., POZZETTI, A., ZORZINI, M. (2006), Production relocation of Italian companies in Romania: an empirical Analysis, Proceedings of the 13th EurOMA Conference 2006, Vol. I, pp. 2 I-30.

CHAKRAVARTY, A., FERDOWS, K. y SINGHAL, K. (1997), Managing international operations versus internationalizing operations management, Production and Operations Management, Vol. 6, No. 2, pp. 100-101.

DEFEE, C. C. y STANK, T. P. (2005), Applying the strategystructure-performance paradigm to the supply chain environment, International Journal of Logistics Management, Vol. 16, No. I, pp. 28-50. 
DE MEYER, A. y VEREECKE, A. (1996), International operations, in Werner, M. (Eds), International Encyclopedia of Business and Management, Routledge, London.

DUBOIS, F.L., TOYNE, B. y OLIFF, M.D. (1993), International manufacturing strategies of U.S. multinationals: a conceptual framework based on a four-industry study, Journal of International Business Studies, Q2 24, 2, pp. 307-333.

FERDOWS, K. ( 1989), Mapping international factory networks, in K. Ferdows (Ed.), Managing International Manufacturing, Elsevier Science Publishers, New York, pp. 3-2I.

FERDOWS, K. (1997), Making the most of foreign factories, Harvard Business Review, March - April, pp. 73-88.

FELDMANN, A. y OLHAGER, J. (2009), Plant roles and decision-making in manufacturing networks, Proceedings 16th annual EurOMA Conference, Gothenburg.

FLAHERTY,T. (1986), Coordinating international manufacturing and technology, in M. Porter (Eds), Harvard Business School Press.

FUSCO, J.P. y SPRING, M. (2003), Flexibility vs. robust networks: the case of the Brazilian automotive sector, Integrated manufacturingsystems, Vol. I4, No. I, pp. 26-35.

GHEMAWAT, P. (20I0), Finding your new strategy in the new landscape, HBR, March.

GOBBO, J., (2007), Inter-firm network: a methodological approach for operations strategy, Proceedings of the I4th EurOMA Conference 2007, Ankara.

HARBISON, F. H., y MYERS, C.A. ( 1989), Management in the industrial world: An international analysis, McGrawHill, New York.

KINRA, A., A., KOTZAB, H., SKJØTT-LARSEN, T. y BAGCHI P.(2007), Developing a Supply Chain competency index for transition economies - an AHP approach, E Proceedings of the 14th EurOMA Conference 2007 conference, Ankara.

KONO,T. (1992), Japanese management philosophy: can it be exported?, Strategic Management in Japanese Companies, Pergamon Press, pp. II-24.

KOONTZ, H. (1969), A model for analyzing the universality and transferability of management, The Academy of Management Journal, Vol. I 2, No.4, pp. 4I 5-429.

KUMON, H., y ABO,T. (2004), The hybrid factory in Europe: The Japanese management and production system transferred, Antony Rowe Ltd., London.

LEE, H. y SILVERMANN, A. (2008), Renault's Logan Car: Managing Customs Duties for a Global Product, HBR, Case Study.

LINSTONE, H.A. y TUROFF, M. ( 1975), The Delphi Method: Techniques and Applications, London Reading, Mass.: Addison-Wesley.
MARITAN, C.A., BRUSH, T.H. y KARNANI (2004), A.G., Plant roles and decision autonomy in multinational plant networks, Journal of Operations Management, Vol. 22, No. 5, pp. 489-503.

MEIJBOOM, B. y VOORDIJK, H. (2003), International operations and location decisions: a firm level approach, Tijdschrift Voor Economische En Sociale Geografie, Vol. 94, No. 4, pp. 463-476.

NETLAND, T., ALFNES, E. y FAUSKE, H. (2007), How mature is your supply chain? - A supply chain maturity assessment test, Proceedings of the 14th EurOMA Conference 2007 conference, Ankara.

OHNO,T. (1 988) The Toyota Production System: Beyond large scale Production. Productivity Press, Portland, Oregon.

PORTER, M.E. (1985), Competitive Advantage, The Free Press, New York.

PRASAD, S., BABBAR, S. y MOTWANI, J. (200I), International operations strategy: current efforts and future directions, International Journal of Operations \& Production Management, 2 I (5/6), pp. 645-665.

RADOVILSKY,Z.D. (1994), Managing operations in the former Soviet Union: current situation and future development, International Journal of Operations \& Production Management, I 4(2), pp. 43-50.

RUDBERG, M. (2004), Linking Competitive Priorities and Manufacturing Networks: A Manufacturing Strategy Perspective, International Journal of Manufacturing Technology and Management, Vol.6, Nos. I/2, pp. 55-80.

SCHMENNER, R.W. (1997), The erosion in European manufacturing, Production and Operations Management, 6 (2), pp. | |0- | 13.

SHI, Y. y GREGORY, M.J. ( 1998), International Manufacturing Networks-to develop global competitive capabilities, Journal of Operations Management,Vol. 16, pp. 195214

SHI,Y.y GREGORY, M.J. (2005), Emergence of global manufacturing virtual networks and establishment of new manufacturing infrastructure for faster innovation and firm growth», Production Planning \& Control, Vol. I6, No. 6, pp. 62I-63I.

SINGH SRAI, J. y SHI,Y. (2008), Understanding China's Manufacturing Value Chain, University of Cambridge, Institute for Manufacturing Publishing, Cambridge.

SLACK, N. y LEWIS, M. (2002), Operations Strategy. Upper Saddle River, NJ: Prentice Hall, 2nd Edition.

SWEENEY, M., COUSENS, A. y SZWEJCZEWSKI, M. (2007), International manufacturing networks design - 
A proposed methodology, Proceedings of the I 4th EurOMA Conference 2007, Ankara.

TEECE, D.J., PISANO, G, y SHUEN, A. ( 1 997) Dynamic capabilities and strategic management, Strategic Management Journal (I 8) 7, pp. 509-533.

TUROFF, M. y HILTZ, S. (1996), Computer based Delphi Processes, http://eies.njit.edu/ turoff/Papers/delphi3. html

VEREECKE, A. y VAN DIERDONCK, R (2002), The Strategic Role of the Plant:Testing Ferdow's Model, International Journal of Operations \& Production Management, Vol. 22, n. 5 .

VEREECKE,A.,VAN DIERDONCK, R. y DE MEYER (2006), A., A typology of plants in global manufacturing networks, Management Science, Vol. 52, No. II, pp. 1737 1750.
VOKURKA, R.J. y DAVIS, R.A.(2004), Manufacturing strategic facility types, Industrial Management and Data Systems, Vol. I 04, No.6, pp. 490-504.

WOMACK, J., JONES, D. y ROOS, D. (1990), The machine that changed the world, Massachusetts Institute of Technology MIT. Macmillan Publishing Company USA.

WOMACK, J y JONES, D.T. (1996), Lean Thinking. Simon \& Schuster, New York.

WONG, C.Y.y GUBI, E. (2007), Managing operations in an expanding Europe - refinement of transnational theory, Proceedings of the 14th EurOMA Conference 2007, Ankara, pp. 426-435.

YOKOZAWA, K., DE BRUIJN, E. J. y STEENHUIS, H.-J. (2007), A conceptual model for the international transfer of the Japanese management systems. Proceedings of the I4th EurOMA Conference 2007, Ankara. 